\title{
Practical Considerations for Integrating PET/CT into Radiation Therapy Planning
}

\author{
Shelley N. Acuff ${ }^{1}$, Anetia S. Jackson ${ }^{2}$, Rathan M. Subramaniam ${ }^{3}$, and Dustin Osborne ${ }^{1}$ \\ ${ }^{1}$ Department of Radiology, University of Tennessee Graduate School of Medicine, University of Tennessee Medical Center, Knoxville, \\ Tennessee; ${ }^{2}$ Department of Radiation Oncology, University of Tennessee Medical Center, Knoxville, Tennessee; and ${ }^{3}$ Division of \\ Nuclear Medicine, Department of Radiology, Department of Clinical Sciences and Harold C. Simmons Comprehensive Cancer \\ Center, University of Texas Southwestern Medical Center, Dallas, Texas
}

Over the past $20 \mathrm{y}$, PET/CT has had many technologic and developmental advancements for patient care. PET/CT has evolved from being used as a diagnosis and staging tool to now having an impact on treating cancer through a collaboration with radiation oncology. There are multiple considerations when integrating $\mathrm{PET} / \mathrm{CT}$ into radiation therapy planning, such as the needs of the PET/CT center, the types of scans to offer, workflow considerations between the PET/CT center and the radiation therapy planning center, $\mathrm{PET/CT}$ center growth and demand on schedules, and the impact PET/CT will have on radiation treatment planning. Careful planning and implementation are important in offering patients optimum care that integrates $\mathrm{PET} / \mathrm{CT}$ in radiation therapy planning.

Key Words: $\mathrm{PET} / \mathrm{CT}$; radiation therapy; radiation oncology; radiation planning; CT therapy planning

J Nucl Med Technol 2018; 46:343-348

DOI: 10.2967/jnmt.118.209452

\section{$\mathbf{S}$} pulmonary nodules in 1998 (1), there has been unprecedented growth and development in technology and clinical applications to benefit patient care. Multiple modalities have come together, including PET with CT and, more recently, PET with MRI to form hybrid PET/CT and PET/ MRI to improve the accuracy of diagnosis, staging, therapy planning, and therapy response assessment. When used correctly, PET/CT can have a great impact on the planning of radiation therapy because of its unique biologic target volumes when compared with other modalities. There are practical considerations to consider in implementing PET/ CT in radiation therapy planning, such as the needs of the PET/CT center, the types of scans to offer, workflow considerations between the PET/CT center and the radiation

\footnotetext{
Received Feb. 6, 2018; revision accepted Jun. 19, 2018.

For correspondence or reprints contact: Shelley Acuff, University of Tennessee Graduate School of Medicine, Molecular Imaging and Translational Research Program, 1924 Alcoa Hwy., Knoxville, TN 37920.

E-mail: sacuff@utmck.edu

Published online Aug. 3, 2018.

COPYRIGHT (c) 2018 by the Society of Nuclear Medicine and Molecular Imaging.
}

therapy planning center, PET/CT center growth and demand on schedules, and the desired impact PET/CT will have on radiation treatment planning (2).

\section{NEEDS OF THE PET/CT CENTER}

The first thing to consider is what needs to be in place for the PET/CT center to offer support for radiation therapy planning. There need to be specific personnel training, hardware requirements, and changes to the PET center schedule to facilitate these studies.

\section{Hardware}

Specific equipment is required to be able to offer the scans needed for radiation therapy planning. Some PET/CT scanners use lower amounts of radiation to produce attention correction-only CT scans and do not produce diagnostic-quality CT scans. To be able to use the PET/CT scanner for radiation therapy, the CT component of the PET/CT scanner needs to be able to produce diagnosticquality scans. Many types of radiation therapy rely on diagnostic-quality CT for planning.

Some radiation therapy planning centers prefer contrastenhanced CT for therapy planning, and a pressure injector will need to be in place to allow the use of intravenous contrast media. Nuclear medicine technologists need to be trained and competent in performing intravenous contrast CT to offer this unique service. Because anaphylactic shock is a risk anytime iodinated contrast agents are used, a physician is needed to cover this responsibility and the staff must be properly trained and prepared in case of an emergency.

A radiation therapy pallet is required for scanning. Therapy tables are flat; therefore, any scans used for therapy planning need to be obtained on a flat surface. The curved contour of a standard PET/CT table may interfere with planning scans, but therapy pallets are designed to slide over the curved PET/CT table to allow scanning on a flat surface that matches the radiation therapy system.

A 4-dimensional infrared patient alignment laser system is needed to assist with correct positioning for the scans. The exact placement of the patient's body on the table is important, and these lasers allow for exact alignment of the patient between imaging table and treatment table (3). 
In addition to exactly aligning the patient's body on the table, placing the actual organ or tissue that is receiving treatment in the correct position is of high importance. An assortment of positioning and stabilizing products is required. Stabilizing products such as face masks, bite blocks, and beanbags are used. Face masks are personally made for each patient using a plastic that when introduced to warm water will mold to the patient's face in the position that is desired. The mask then dries to a hard, firm structure that will place the patient's head in exactly the same position every time (Fig. 1). For example, for treatment of a head or neck tumor, the use of a face mask and bite block, which are molded in the same way to fit each individual patient, may be required to prevent motion and to position the tongue (3). If the patient were to experience some form of anatomic change such as surgery or excessive weight loss, a new mask would need to be made $(4,5)$. Beanbag stabilizers are used by subtracting air from the bag to create a mold around specific areas of the body to prevent motion during imaging and treatment and are personally made for each patient as well (Fig. 1) $(3,6)$.

Lastly, computers will need to be in place to allow for the complex task of the integrating the images, such as sending images from the PET/CT system to the radiation therapy system. Multiple computer workstations are available on the market for this use. These workstations not only are required but also allow for the option of a collaborative method by which either department can offer regions of interest or SUVs.

\section{Scheduling}

Once the PET/CT center has all essential hardware and trained personnel, it is time to look at the schedule. There need to be dedicated time slots on the PET/CT schedule to support radiation therapy scans. Most of the scans will

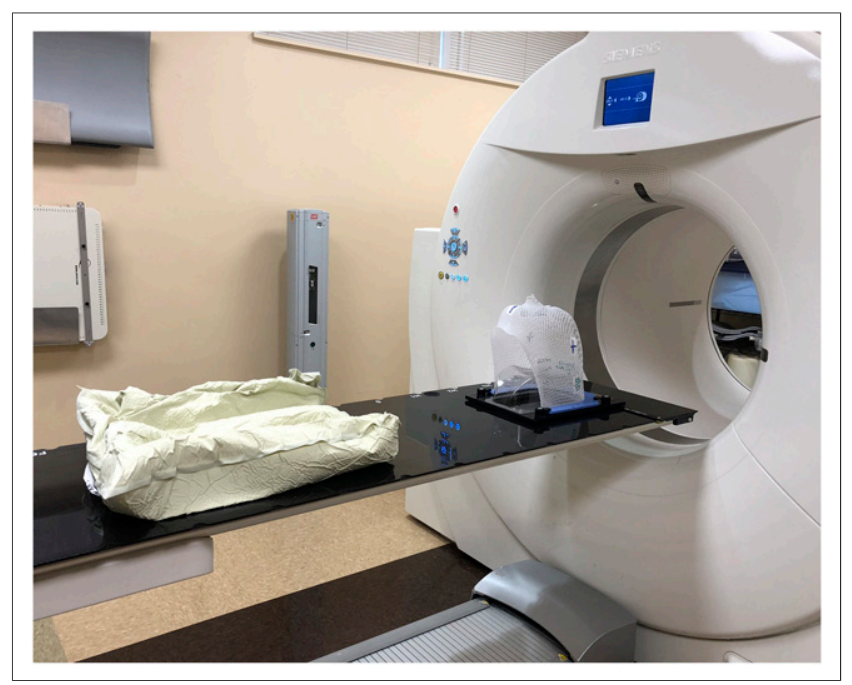

FIGURE 1. Radiation pallet placed on PET/CT table with face mask and beanbag stabilizer. Four-dimensional laser system is also seen. This is what a common setup looks like when using PET/CT system to perform radiation therapy imaging. require scheduling PET imaging and radiation therapy resources so they can be available at the same time. A strong communication channel needs to be in place between the PET center, radiation oncology center, and scheduling team to prevent logistical errors.

Typically, therapy scans take $15-30 \mathrm{~min}$ of table time, depending on the body region being imaged. This timing makes therapy scans compatible with most PET/CT time slots, which are $30 \mathrm{~min}$. Occasionally, a patient may need a longer time slot because of a specific type of patient prep.

Once the radiation oncologists and therapists are collaborating, they should jointly discuss with the referring physician the need to order the PET/CT scan and the radiation therapy planning scan at the same time so that the two can be combined, which produces the best images for planning therapy. Combining the two will also be helpful to patients by allowing them to undergo the 2 scans on the same day instead of traveling to the center on 2 separate days.

\section{SCANS TO OFFER}

With appropriate equipment and communication channels in place, it is possible to support radiation oncology with multiple imaging services using a PET/CT imaging system. For initial therapy planning, routine follow-up, and disease progression, a standard whole-body PET/CT scan is used. Once the radiation oncologists use the standard whole-body PET/CT for initial treatment planning, then disease progression will be monitored using follow-up whole-body PET/CT scans.

A diagnostic-quality CT therapy planning scan can be offered on the PET/CT system and used to plan a patient's initial radiation treatment. For this scan, a combination team of a PET/CT technologist and a radiation therapist is needed. The PET/CT technologist will facilitate operating the PET scanner, whereas the radiation therapist will set the patient up with the appropriate restraints, stabilizing products, and positioning.

For lesions in an area with a lot of motion, a 4dimensional gating scan using CT or PET can be applied to accurately assess motion and changes in fiducial marker movement over the course of therapy. Depending on the type of treatment the patient will receive, tumor motion can be a big factor. Specific types of radiation planning benefit from seeing the full range of motion of the tumor and other areas that are involved due to the motion. Fiducial marker placement and the movement of the fiducials are important for treatment planning. For accurate treatment, the fiducials need to be in the accurate place and move in the same motion as the tumor.

If it known with certainty that a patient entering the outpatient imaging workflow will be treated at the same institution, offering a combined PET/CT and CT radiation therapy planning study is a great option. The patient's PET/ CT scan and CT radiation therapy scan will be scheduled on the same day in consecutive time slots. The workflow starts 
with the PET/CT scan. At the end of the PET/CT scan, the radiation therapist positions the patient using the face mask or any other stabilizing products needed and completes the $\mathrm{CT}$ radiation therapy planning scan. Immediately afterward, an additional PET image in the same position can be completed over the area of interest. This workflow offers a standard whole-body PET/CT scan for initial treatment planning, a CT scan for planning radiation therapy, and a PET image of only the area of interest that spatially matches the $\mathrm{CT}$ planning scan.

\section{PET/CT CENTER GROWTH}

Once the collaborative workflow begins, there may be an increase in PET/CT requests from referring physicians and radiation oncologists, requiring additional logistics considerations for outpatient PET/CT imaging. Discussions with the radiation oncology group when the collaboration is being developed should include determining the expected number of referrals and the anticipated growth of radiation oncology. These projections should be considered in planning imaging services, staffing, and scheduling to handle the increased load without creating unacceptable wait times. Although the cost for the needed hardware, equipment, and new technology will vary and can be expensive, the PET/CT growth that comes from this type of collaboration can, over time, absorb that cost and even help bring in more profit for the PET/CT center. Our firstyear collaboration results indicate the potential growth that can be observed with such collaborations.
In the first year of radiation oncology PET/CT collaborations, radiation oncologists directly added 108 PET/CT procedures to our outpatient workflow. During the first half of the year, 40 PET/CT procedures were ordered, with 68 added in the second 6-mo period. Over the course of the first year, we experienced growth of $70 \%$ from the first half of the year to the second, thus suggesting the potential growth capabilities of this type of collaboration. These data do not include any indirect referrals that came from other oncology groups that may have been influenced by consults with radiation oncology.

As new software and imaging technology are added to the PET/CT system, it is important to inform the radiation oncology group on how that technology is used in imaging and discuss how the new technology might be used with radiation therapy planning. Technologies that are specific to radiology and PET/CT imaging may not be familiar to radiation oncology teams. Having discussions and educational reviews on new features will assist with their adoption and may improve image quality or increase the efficiency of imaging workflows for both radiology and radiation oncology services.

Another recent potential growth area for PET/CT and radiation oncology collaborations is the use of newly approved radiopharmaceuticals for PET imaging. ${ }^{18}$ F-FDG has been the primary radiopharmaceutical used to diagnose, stage, and monitor cancer in patients by imaging glucose utilization in the body. Within the last $2 \mathrm{y}$, new radiopharmaceuticals for imaging neuroendocrine tumors and prostate

TABLE 1

Differences in Efficiency of Radiation Therapy Workflow With Versus Without PET/CT for Planning

\begin{tabular}{|c|c|c|c|c|c|c|c|}
\hline \multicolumn{4}{|c|}{ Workflow for initial treatment } & \multicolumn{4}{|c|}{ Workflow for prior treatment } \\
\hline \multicolumn{2}{|c|}{ Without PET/CT } & \multicolumn{2}{|c|}{ With PET/CT } & \multicolumn{2}{|c|}{ Without PET/CT } & \multicolumn{2}{|c|}{ With PET/CT } \\
\hline Task & Time (min) & Task & Time (min) & Task & Time (min) & Task & Time (min) \\
\hline Import images & 3 & Import images & 3 & Import images & 3 & Import images & 3 \\
\hline Image registration* & 20 & Image registration $^{\dagger}$ & 3 & Image registration ${ }^{\star}$ & 20 & Rigid registration & 10 \\
\hline \multirow[t]{5}{*}{ Verify registration } & 20 & Verify registration & 4 & Verify registration & 20 & Verify registration & 20 \\
\hline & & & & $\begin{array}{l}\text { Transfer/deform } \\
\text { contours }\end{array}$ & 30 & Transfer contours & 10 \\
\hline & & & & $\begin{array}{l}\text { Verify contours on } \\
\text { new CT }\end{array}$ & 10 & $\begin{array}{l}\text { Verify contours on } \\
\text { new CT }\end{array}$ & 10 \\
\hline & & & & $\begin{array}{l}\text { Deform prior dose } \\
\text { to current CT }\end{array}$ & 10 & & \\
\hline & & & & $\begin{array}{l}\text { Verify dose transfer } \\
\text { to new CT }\end{array}$ & 10 & & \\
\hline $\begin{array}{l}\text { Import images into } \\
\text { planning software }\end{array}$ & 4 & & & $\begin{array}{l}\text { Import images into } \\
\text { planning software }\end{array}$ & 4 & & \\
\hline Contour & 240 & Contour & 120 & Contour & 240 & Contour & 240 \\
\hline \multirow[t]{3}{*}{ Planning } & 1,440 & Planning & 1,440 & Planning & 1,440 & Planning & 1,440 \\
\hline & & & & Insert plan sum & 5 & Insert plan sum & 5 \\
\hline & & & & $\begin{array}{l}\text { Evaluate plan } \\
\text { summation }\end{array}$ & 10 & $\begin{array}{l}\text { Evaluate plan } \\
\text { summation }\end{array}$ & 10 \\
\hline Total time & 1,727 & Total time & 1,570 & Total time & 1,802 & Total time & 1,748 \\
\hline
\end{tabular}

*Deformable registration required.

†Native registration. 


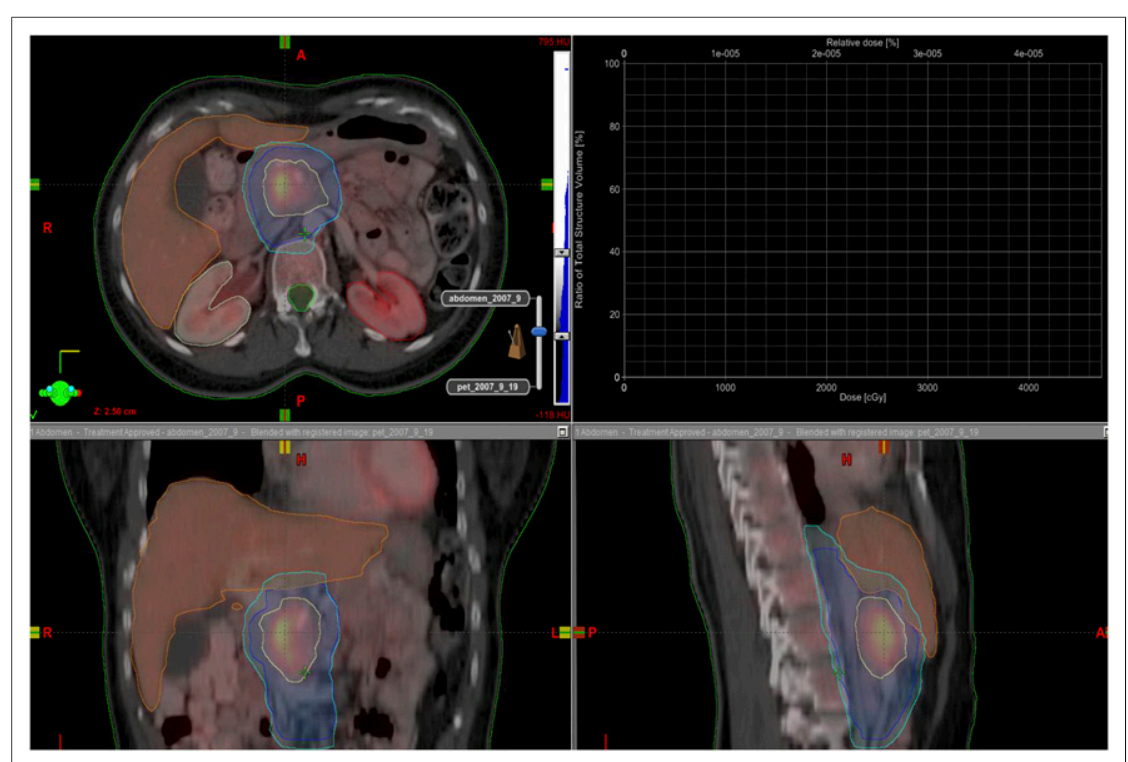

FIGURE 2. Abdominal mass example in which PET/CT imaging was used to aid in drawing treatment-planning contours. ${ }^{18} \mathrm{~F}-\mathrm{FDG}$ uptake visualized by PET/CT is used to determine gross tumor volume, shown in yellow. Area in dark blue is clinical tumor volume, defined as gross tumor plus microscopic disease. Cyan outline is planning target volume, which includes clinical tumor volume plus margin for daily treatment setup and patient positioning.

lesions have been approved for use in the United States, with more specific tumor-seeking agents on the horizon. Access to these compounds will create additional opportunities for collaborative PET-radiation oncology services and provide more individualized imaging and radiation therapy planning.

\section{IMPACT OF PET/CT ON RADIATION TREATMENT}

The collaboration between PET/CT and radiation oncology allows PET/CT imaging to have an impact on diagnosis, staging, treatment care path, target delineation, clinical trials, research, and follow-up. Treatment planning for a patient begins with the physicians discussing the diagnosis and staging aspect, typically as part of tumor

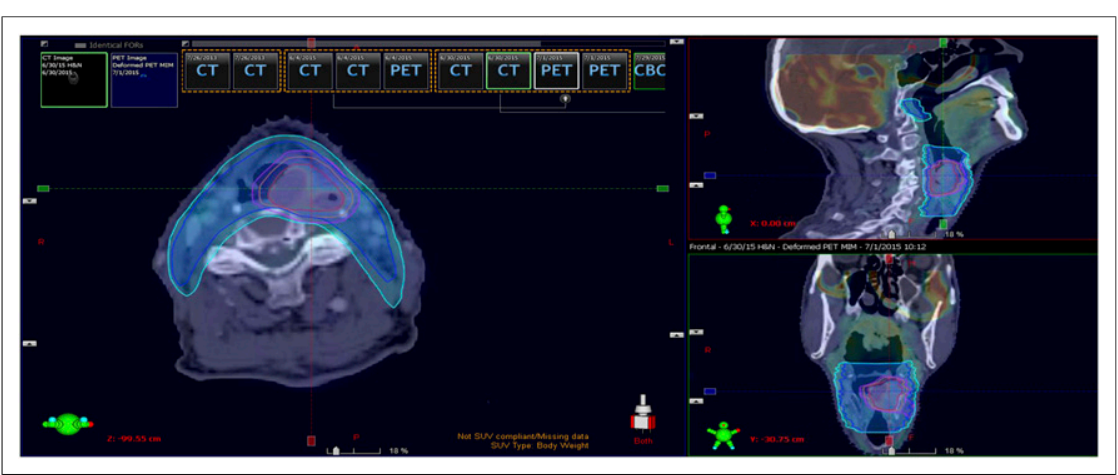

FIGURE 3. Example of intratreatment use of PET/CT imaging for head-and-neck patient who presented with initial disease. Because of weight loss, patient had to have new face mask made and follow-up CT. Original PET/CT images were registered to new CT images, allowing patient to continue treatment without any breaks. board discussions. To stage a patient, a radiation oncologist will use PET/CT to determine the gross tumor volume, metastatic disease, or nodal involvement. The physician will discuss with the medical oncologist, surgical oncologist, and other physicians the best treatment plan for the patient involving chemotherapy and radiation; this is referred to as the treatment care path. If radiation therapy is prescribed, the physician can immediately begin the process of planning the treatment and will delineate the target treatment volume. The particular performance status or disease involvement in some patients make palliative care the best option for overall outcome.

PET/CT collaboration may improve the treatment planning workflow for patients who are receiving their initial treatment and for patients who have had prior radiation treatment. Use of an integrated PET/CT radiation therapy workflow results in similar patient positioning between modalities, enabling several key benefits. For example, rigid registration between modalities can be used instead of the more error-prone deformable registration. Lesion contours can be more easily transferred, reducing inaccuracies in radiation therapy plans. Evaluation of the dose with respect to critical structure and tumor volume can be completed more rapidly, leading to a more efficient process for treatment planning by reducing the registration time, fusion review time, and contouring time. Exposure of the patient to radiation can be reduced because a single-scan workflow can be used. Finally, robust collaboration between radiation oncology and PET/CT imaging services can enable a more efficient and effective pretreatment and treatment planning process.

Table 1 summarizes the differences in efficiency that may be realized in the radiation therapy workflow when $\mathrm{PET} / \mathrm{CT}$ imaging is incorporated versus not incorporated for therapy planning. With collaboration, times for initial and prior treatment are improved, with maximum time savings of approximately $157 \mathrm{~min}$ and $54 \mathrm{~min}$, respectively.

The clinical relevance of imaging and therapy collaborations for radiation oncology patients can be appreciated by reviewing several clinical examples. Three cases are presented below: a pretreatment case, an intratreatment case, and a posttreatment case. The institutional review board did not require 


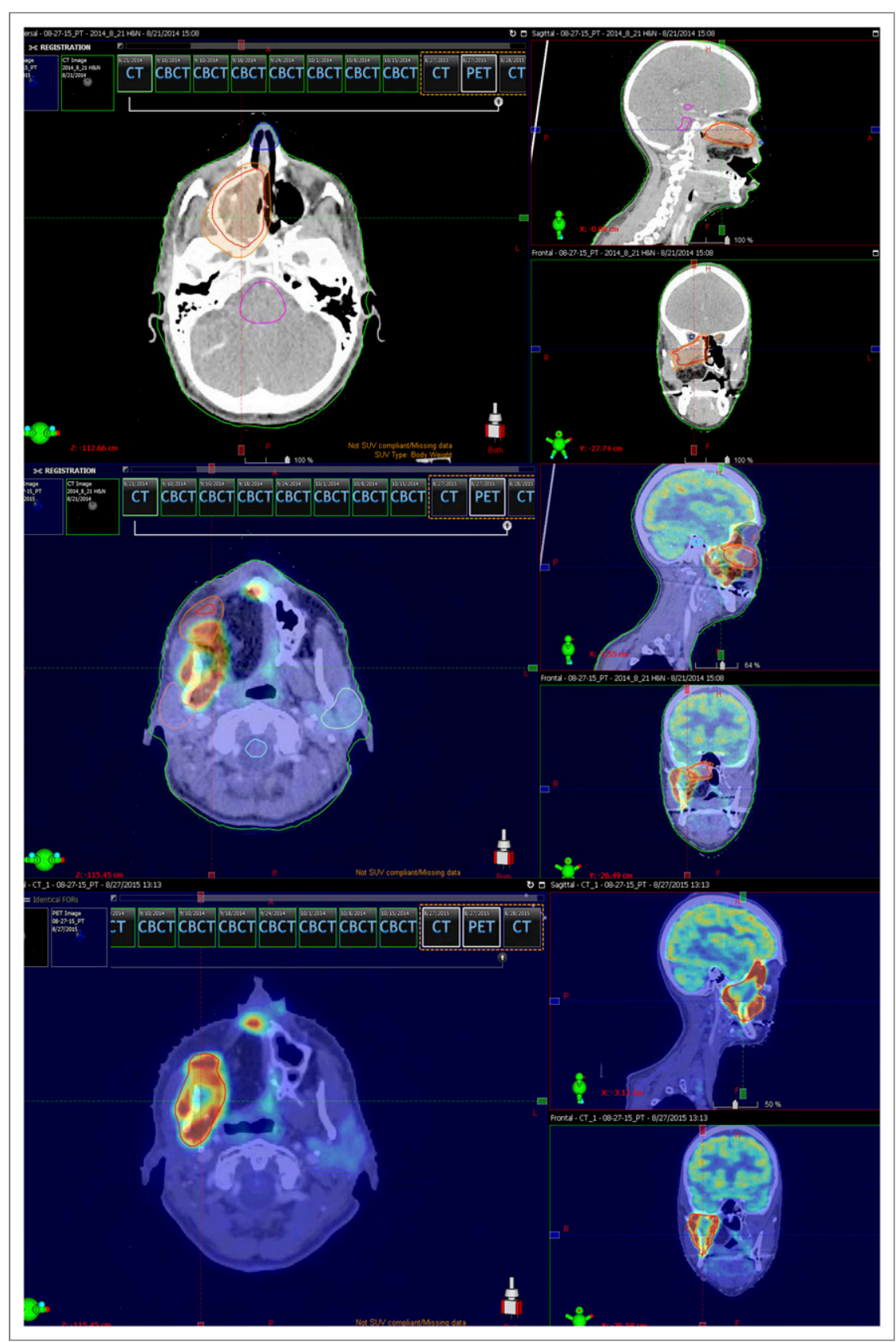

FIGURE 4. Example head-and-neck patient, with top images showing pretreatment volumes, middle images showing 1-y PET/CT follow-up, and bottom images showing new treatment volumes while using PET/CT image

consent from these patients because the images were being used for educational case study purposes.

\section{Pretreatment Case}

In the pretreatment example (Fig. 2), PET/CT imaging was used to aid in drawing treatment planning contours for a patient with an abdominal mass. A typical planning workflow would define 3 primary volumes: gross tumor volume, clinical tumor volume, and planning target volume (7). Gross tumor volume when using PET describes the extent of disease visible on imaging. In this example, the ${ }^{18} \mathrm{~F}-\mathrm{FDG}$ uptake visualized by PET/CT was used to determine the gross tumor volume.

The clinical tumor volume is the area included in the gross tumor volume plus some additional margin related to subclinical microscopic disease. This volume is highly variant and complex to determine, as the disease extent cannot be accurately measured.

The planning target volume included the clinical tumor volume plus a margin for daily treatment setup and patient positioning. The purpose of a properly defined planning target volume is to make sure that the defined clinical tumor volume receives the appropriate radiation dose prescription. These volumes are used in defining the patient treatment plan.

\section{Intratreatment Case}

In the intratreatment example (Fig. 3 ), a head-and-neck patient who presented with initial disease, the therapy path was planned using PET/CT with a mask molded to fit the patient to increase positional accuracy between the imaging and therapy systems. During treatment, the patient lost weight, requiring a new setup and the creation of a new face mask. For this study, the patient's previous PET/CT scan was registered to the follow-up CT scan using deformable registration. This methodology is used to determine adjusted treatment volumes quickly, as previous contours can be deformed to match contours on the new imaging studies. With this methodology, the patient was able to continue the full course of radiation without the need to build in any breaks.

\section{Posttreatment Case}

The posttreatment example (Fig. 4) was another head-and-neck case. A 1-y posttreatment PET/CT study was fused to the pretreatment study, allowing delineation of the new treatment volumes. Knowledge of the previous treatment volume and contour placement allowed treatment of a reoccurring tumor next to the prior treatment volume.

\section{CONCLUSION}

Collaboration between a PET/CT center and a radiation oncology center requires consideration of multiple factors, such as the needs of the PET/CT center, the types of scans 
to offer, the workflow between the 2 centers, PET/CT center growth and demand on schedules, and the impact PET/CT will have on radiation treatment planning. Imaging and therapy collaborations will contribute to a better course of treatment, increased efficiency in workflow and time management within the centers, and positive effects on the pretreatment, intratreatment, and posttreatment of radiation oncology patients.

\section{DISCLOSURE}

No potential conflict of interest relevant to this article was reported.

\section{ACKNOWLEDGMENT}

The cost to print this paper was funded by the generosity of the PET Center of Excellence through an SNMMI Councils/Centers Internship.

\section{REFERENCES}

1. Medicare Coverage Issues Manual: change request 1603-section 50-36, positron emission tomography (PET) scans. Centers for Medicare and Medicaid Services website. https://www.cms.gov/Regulations-and-Guidance/Guidance/Transmittals/ downloads/R136CIM.pdf. Published April 10, 2001. Accessed September 17, 2018.

2. Acuff S, Jackson S, Osborne D. Clinical considerations for using PET/CT in radiation therapy [abstract]. $J$ Nucl Med. 2016;57(suppl 1):2647.

3. Verhey LJ, Goitein M, McNulty P, Munzenrider J, Suit H. Precise positioning of patients for radiation therapy. Int J Radiat Oncol Biol Phys. 1982;8:289294.

4. Schoenfeld JD, Kovalchuk N, Subramaniam RM, Truong MT. PET/CT of cancer patients: part 2, deformable registration imaging before and after chemotherapy for radiation treatment planning in head and neck cancer. AJR. 2012;199:968-974.

5. Kovalchuk N, Jalisi S, Subramaniam RM, Truong MT. Deformable registration of preoperative PET/CT with postoperative radiation therapy planning CT in head and neck cancer. Radiographics. 2012;32:1329-1341.

6. Bentel GC, Marks L, Sherouse G, Spencer D, Anscher M. The effectiveness of immobilization during prostate irradiation. Int J Radiat Oncol Biol Phys. 1995;31: 143-148.

7. Burnet NG, Thomas SJ, Burton KE, Jefferies SJ. Defining the tumor and target volumes for radiotherapy. Cancer Imaging. 2004;4:153-161. 